Research Article

\title{
Pricing Vulnerable Options in the Bifractional Brownian Environment with Jumps
}

\author{
Panhong Cheng $\mathbb{D}^{1,2}$ and Zhihong $\mathrm{Xu} \mathbb{D}^{3}$ \\ ${ }^{1}$ Business School, University of Shanghai for Science and Technology, Shanghai 200093, China \\ ${ }^{2}$ Institute of Mathematics and Finance, Chuzhou University, Chuzhou 239000, China \\ ${ }^{3}$ Public Teaching Department, Rizhao Polytechnic, Rizhao 276826, China \\ Correspondence should be addressed to Panhong Cheng; hollycheng@sina.cn
}

Received 27 August 2021; Accepted 18 October 2021; Published 2 November 2021

Academic Editor: Antonio Di Crescenzo

Copyright (c) 2021 Panhong Cheng and Zhihong Xu. This is an open access article distributed under the Creative Commons Attribution License, which permits unrestricted use, distribution, and reproduction in any medium, provided the original work is properly cited.

\begin{abstract}
In this paper, we study the valuation of European vulnerable options where the underlying asset price and the firm value of the counterparty both follow the bifractional Brownian motion with jumps, respectively. We assume that default event occurs when the firm value of the counterparty is less than the default boundary. By using the actuarial approach, analytic formulae for pricing the European vulnerable options are derived. The proposed pricing model contains many existing models such as Black-Scholes model (1973), Merton jump-diffusion model (1976), Klein model (1996), and Tian et al. model (2014).
\end{abstract}

\section{Introduction}

Options are popular financial derivatives that play important roles in financial markets. An option is a financial contract that the option holder has the right to buy or sell a certain amount of underlying assets to the option issuer (the counterparty) at an agreed strike price, but the holder does not have the obligation to buy or sell the underlying assets. There is no free lunch in the world. Due to the asymmetry of right and obligation in an option trading, the holder must pay a certain amount that is called options price to the issuer in order to obtain the right given by the contract. Reasonable options price is the premise of the trading between the holder and the issuer, and it is not only a favorable guarantee for investors to hedge and make profit but also of great significance to improve the theoretical system of options pricing. Therefore, options pricing has always been received great attention by many researchers.

Black and Scholes [1] presented the famous Black-Scholes formulae based on Fama's efficient market hypothesis [2]. The pricing model proposed by Black and Scholes marks the establishment of modern options theory. Since this pricing model is based on a pure diffusion process, the process for the underlying stock prices may have the effect of understating the valuation of options in many business situations, using jumps to capture sudden shocks occurred in the underlying asset prices which are necessary and this can make options pricing more accurate. Merton [3] first incorporated jump risk into options pricing model and proposed a jump-diffusion model that effectively improved the Black-Scholes model. Since then, many researchers have further studied the pricing problem of options. For example, Jarrow and Rosenfeld [4] and Ball and Torous [5] observed that the underlying asset prices exhibited lognormal jumps. Kou [6] divided the stock price into continuous and discontinuous parts and proposed a double exponential jumpdiffusion model with the assumption that the jump amplitude of discontinuous part obeyed double exponential distribution. Rodrigo [7] studied the pricing problem of barrier options where the underlying asset prices were governed by geometric Brownian motion with jumps and obtained explicit pricing formulae for barrier options. More studies about options pricing with jump-diffusion processes could be seen in reference [8-11] and the references therein.

All studies mentioned above mostly focused on the options pricing problem without credit risk. When options 
trading activities have been moving from standardized products quoted on exchange-traded markets towards customized products traded on the over-the-counter (OTC) markets, OTC derivatives market cannot guarantee that the counterparty realizes the promise of payments to the option holder. Thus, the credit risk which is also called default risk exists in the OTC options trading, especially due to the frequent bankruptcy of domestic and foreign companies during the financial crisis, such as the sudden bankruptcy of Lehman Brothers after the harrowing experience of the subprime crisis in 2007. The issue of credit exposure has attracted much attention when pricing financial derivatives in the OTC market. Recently, OTC derivatives market has grown rapidly. In 2020, the notional amounts outstanding of the OTC options contract reached 107.4 trillion dollars based on the bank for International Settlements (in short, BIS) statistics on OTC derivatives outstanding. So, it is necessary to consider the potential credit risk caused by the counterparty when studying the options pricing in OTC market. The options with credit risk are defined as vulnerable options. As for the vulnerable options pricing, it is important to characterize the credit risk of the counterparty. Currently, there are two basic approaches in the existing literature that capture the credit risk; one is the structural approach. Merton [12] first proposed the structural model, which assumed that when the firm value of the counterparty was less than the default boundary, the counterparty would not realize the promise of payments to the option holder, that is, default occurred. The other is the reduced-form approach. Jarrow and Turnbull [13] introduced this model, which provided a new framework to model the default time and defined the first jump time as the default time. The default probability was controlled by a default intensity. Based on these two approaches, many researchers have studied the pricing problem of vulnerable options. Johnson and Stulz [14] first put forward the vulnerable options pricing model assuming that the options were the only liability of the options issuer and the underlying asset prices and the firm values of the counterparty were independent. Under the structural model, Klein [15] extended the model of Johnson and Stulz [14] by allowing the options issuer to have other liabilities and extended the results of Jarrow and Turnbull [13] by relaxing the independence assumption. Contrast to constant interest rate assumption in [15], Klein and Inglis [16] considered the valuation of vulnerable options with stochastic interest rate and derived an exact pricing formula of vulnerable option through partial differential equation techniques. In order to describe the phenomenon of volatility clustering and volatility smile, Lee et al. [17] incorporated Heston stochastic volatility model to capture the behavior of underlying asset prices and firm values of the counterparty and provided a closed-form analytic formula of vulnerable options price by using the Green function and Fourier transforms. Taking jump risk of financial assets into account, Tian et al. [18] investigated the pricing problem of vulnerable options where both of the underlying asset price and firm value of the counterparty followed jump-diffusion processes and jumps in these processes which were divided into idiosyncratic jumps and common jumps besides diffusion parts and got explicit formulae of vulnerable option prices by using the theory of stochastic analysis. Wang [19] extended the framework of Tian et al. [18] to value vulnerable options when default barriers were stochastic. Since vulnerable options may face not only the jump risk but also the illiquidity risk, Yang et al. [20] constructed a pricing model for the European vulnerable options under incomplete information and discussed the impact of pricing parameters on option prices by numerical experiments. Niu et al. [21] studied the valuation of European vulnerable options where the underlying asset price was driven by a double exponential jump-diffusion process and the credit risk was described by the reducedform approach and obtained the pricing formulae of vulnerable options via the equivalent martingale measure technique and two-dimensional Laplace transform. More valuation of vulnerable options could be found in references [22-30] and the references therein.

All the pricing models mentioned above were established under the efficient market hypothesis, which assumed that the logarithmic returns of the financial assets obeyed the standard Brownian motion. But this treatment is contrasted with a series of financial empirical studies that show financial asset prices have many characteristic properties such as high peaks, heavy tails, periods of constant values [31], and longrange dependence [32-34]. Therefore, many generalizations of the celebrated Black-Scholes model are studied. For example, in order to describe properly financial data exhibiting periods of constant values, Magdziarz [35] introduced the subdiffusive geometric Brownian motion (in short, SGBM) as a model of financial asset prices exhibiting subdiffusive dynamics. Then, the author obtained the fractional Fokker-Planck equation (in short, FFPE) governing the dynamics of the probability density function of the introduced process and the pricing formula for a $\mathrm{Eu}$ ropean option in subdiffusive regime. This FFPE was first derived from the continuous-time random walks scheme with heavy-tailed waiting times (periods when the asset prices stay motionless) [36-40]. According to the distribution characteristics of waiting times, Magdziarz and Gajda [41] proposed a generalization of Black-Scholes model which was defined as standard GBM, subordinated by the infinitely divisible inverse subordinator. Since the waiting times could be chosen from the rich family of nonnegative infinitely divisible distributions, the proposed model was an extension of the model in [35]. The corresponding FFPE and formulae for fair prices of European options were obtained. Motivated by subdiffusive motion of the particle observed in biophysics, Jeon and Metzler [42] studied the stochastic properties of a non-Brownian particle whose motion is governed by either fractional Brownian motion (in short, FBM) or the fractional Langevin equation in combined geometries. FBM has been extensively used in the various fields, including but not limited to biology, physics, economics, and so on. Peter [43] put forward the fractal market hypothesis, analyzed different capital markets by using R/S method, and observed the existence of fractal structure and aperiodic cycle. That is to say that the financial asset prices depend not only on the current situation but also on the 
historical moment. Thus, FBM that exhibits properties of self-similarity and long-range dependence has the ability to capture the behavior of financial asset prices. Although FBM had two important properties, Rogers [44] and Cheridito [45] discovered that there existed arbitrage opportunities when FBM was applied to financial markets. Björk and Hult [46] showed that the application of FBM based on Wick integral in financial market was limited.

To overcome these problems and take the long-range dependence of financial asset prices into consideration, many researchers began to use a bifractional Brownian motion to describe the dynamics of financial asset prices. The bifractional Brownian motion (in short, BFBM), known as a generalization of FBM, is a family of centered Gaussian processes that not only has the characteristics of self-similarity and long-range dependence but also has the property of nonstationary increment and can be a semimartingale under appropriate conditions, and then it can be applied to model the evolution of financial asset prices such as the underlying stock prices in options pricing. The BFBM was introduced by Houdre and Villa [47]. Russo and Tudor [48] studied several properties of the BFBM. Es-Sebaiy and Tudor [49] gave the stochastic integral of the BFBM and illustrated that the BFBM was a valuable stochastic process to capture dynamics of financial asset prices. Lifshits and Volkova [50] considered the existence of the BFBM for a given pair of parameters. $\mathrm{Xu}$ [51] proposed a pricing model for European options and compound options in the BFBM environment and derived the pricing formulae by the method of variable substitution and risk-neutral principle. More discussions of the BFBM could be seen in references [52-56] and the references therein.

In general, the valuation of options is derived by using some techniques such as equivalent martingale measure transform, partial differential equation, and Mellin transform. But, there are some assumptions for constructing the options pricing model before using these techniques, and the complexity of the calculation exists as appeared in these techniques. Therefore, an actuarial approach, which does not consider economic assumptions of financial markets and is not only valid in the arbitrage-free, equilibrium, and complete markets but also reliable in the arbitrage, nonequilibrium, and incomplete markets, is a very good method to solve the options pricing model. The actuarial approach proposed by Bladt and Rydberg [57] transformed the options pricing problem into an equivalent fair insurance premium problem. Yan et al. [58] obtained the pricing formulae of European options in the case where the underlying asset price followed Ornstein-Uhlenbeck process by actuarial approach. Falkowski [59] proposed a fractional Black-Scholes pricing model with time-dependent volatility for options and derived the analytic formulae via an actuarial method. Shokrollahi and Klllçman [60] studied the strategy of fair insurance premium actuarial approach for pricing foreign currency option under the jump mixed fractional Brownian motion environment. A lot of the literature on options pricing using the actuarial approach could be found in $[61-64]$.
Despite several literatures having considered vulnerable options pricing, applying bifractional Brownian motion with jumps to model vulnerable options pricing has not been studied. This paper is devoted to the valuation of European vulnerable options in the BFBM environment with jumps, and the structural approach is chosen to model the credit risk. Specifically, the dynamics of the underlying asset prices and firm values of the counterparty are both generated by a two-part stochastic process: one is the continuous price movements generated by a BFBM. The other one is discontinuous changes generated by a Poisson process due to sudden shocks. Following Tian et al. [18], the discontinuous changes in the underlying asset prices and firm values of the counterparty both consist of two parts: idiosyncratic shocks for each asset price and common ones that have an impact on all asset prices. Then, we show how to price European vulnerable options by actuarial approach in a jump bifractional Brownian environment. After obtaining the pricing formulae, the relationship between our pricing formulae and those by Black and Scholes [1], Merton [3], Klein [15], and Tian et al. [18] are discussed.

The rest of our paper is organized as follows. Section 2 provides some preliminaries about the BFBM and actuarial approach. In Section 3, a pricing model for vulnerable options in the jump BFBM environment based on the structural approach is proposed, and the pricing formulae are derived by actuarial approach. Finally, we give the concluding remarks in Section 4.

\section{Preliminaries}

In this section, we present some definitions and results that are needed for the rest of this paper.

\subsection{Bifractional Brownian Motion}

Definition 1. A bifractional Brownian motion $B^{H, K}=\left(B_{t}^{H, K}\right)_{t \geq 0}$ with Hurst parameter $H \in(0,1)$ and $K \in(0,1]$ is a centered Gaussian process with the mean zero and covariance function

$$
\begin{aligned}
R_{H}(t, s) & :=E\left(B_{t}^{H, K} B_{s}^{H, K}\right) \\
& =\frac{1}{2^{K}}\left(\left(t^{2 H}+s^{2 H}\right)^{K}-|t-s|^{2 H K}\right), \quad s, t \geq 0,
\end{aligned}
$$

if $K=1, B^{H, K}$ is the ordinary fractional Brownian motion with Hurst parameter $H \in(0,1)$. Moreover, when $H=(1 / 2), B^{H, K}$ is the standard Brownian motion.

Now, recalling the results in references $[48,52,53]$, some properties of the BFBM are summarized as follows:

(i) For $t \geq 0, E\left(B_{t}^{H, K}\right)^{2}=t^{2 H K}$.

(ii) The BFBM is a self-similar process with Hurst parameter $H K$, that is,

$$
\left\{B_{\alpha t}^{H, K}, t \geq 0\right\} \stackrel{d}{=}\left\{\alpha^{H K} B_{t}^{H, K}, t \geq 0\right\}, \quad \alpha>0,
$$


where $X \stackrel{d}{=} Y$ means the two processes $X$ and $Y$ have the same distributions.

(iii) If $H K>(1 / 2)$, the BFBM exhibits long-range dependence in the case $\sum_{n=1}^{\infty} \rho(n)=+\infty$, where

$$
\rho(n)=E\left[\left(B_{1}^{H, K}\right)\left(B_{n+1}^{H, K}-B_{n}^{H, K}\right)\right] .
$$

(iv) For every $s, t \geq 0$, we have

$$
2^{-K}|t-s|^{2 H K} \leq E\left(B_{t}^{H, K}-B_{s}^{H, K}\right)^{2} \leq 2^{1-K}|t-s|^{2 H K} .
$$

Note that $B^{H, K}$ does not have stationary increments, except in the case $K=1$, and $B^{H, K}$ has $(H K-\delta)$-Hölder continuous paths for any $\delta \in(0, H K)$, thanks to Kolmogorov's continuity criterion.

2.2. Actuarial Approach. The payoff of a European vulnerable call option and put option at maturity date $T$ in this paper are expressed which are consistent with the reference [15] by the following equations, respectively,

$$
\begin{aligned}
& C_{T}^{d}=E\left[\left(S_{T}-X\right)^{+}\left(I_{\left\{V_{T} \geq D^{*}\right\}}+\frac{1-\alpha}{D} V_{T} I_{\left\{V_{T}<D^{*}\right\}}\right)\right], \\
& P_{T}^{d}=E\left[\left(X-S_{T}\right)^{+}\left(I_{\left\{V_{T} \geq D^{*}\right\}}+\frac{1-\alpha}{D} V_{T} I_{\left\{V_{T}<D^{*}\right\}}\right)\right],
\end{aligned}
$$

where $S_{T}$ and $V_{T}$ are the stock price and the firm value of the counterparty at maturity date $T$, respectively. $X$ is the strike price of the options. $D^{*}$ is a constant default boundary such that no credit loss occurs if $V_{T}$ is greater than $D^{*}$ and the option holder receives full payments. If $V_{T}$ is less than $D^{*}$, the option issuer cannot realize the promise of all payments to the holder, then the credit loss occurs. In the event of the credit loss, the option holder only obtains the proportion $((1-\alpha) / D) V_{T}$ of the nominal claim by the option issuer, where $\alpha$ denotes the deadweight costs associated with bankruptcy expressed as a percentage of the firm value of the counterparty and $D$ is the value of total liabilities given by $D^{*}$ and an additional liability.

Definition 2. Assume that $\beta_{S}(w)$ and $\beta_{V}(w)(w \in[0, T])$ are the expected yield rates of the stock price process $\left\{S_{t}, t \geq 0\right\}$ and the firm value process of counterparty $\left\{V_{t}, t \geq 0\right\}$ on $t \in[0, T]$, respectively, which are defined as follows:

$$
\begin{aligned}
& \frac{E\left(S_{T}\right)}{S_{0}}=\exp \left(\int_{0}^{T} \beta_{S}(w) \mathrm{d} w\right), \\
& \frac{E\left(V_{T}\right)}{V_{0}}=\exp \left(\int_{0}^{T} \beta_{V}(w) \mathrm{d} w\right) .
\end{aligned}
$$

Definition 3. Suppose that $C_{0}^{d}$ and $P_{0}^{d}$ are the value of the European vulnerable call and put option at time 0 with the maturity date $T$, respectively, whose stock price is $S_{t}$, firm value of the counterparty is $V_{t}$, and the strike price is $X$. Then, the value of the European vulnerable options by actuarial approach is presented as follows, respectively:

$$
\begin{aligned}
& C_{0}^{d}=E\left[\left(e^{-\int_{0}^{T} \beta_{S}(w) \mathrm{d} w} S_{T}-e^{-r T} X\right)\left(I_{A_{1}, B_{1}}+\frac{1-\alpha}{e^{-r T} D} e^{-\int_{0}^{T} \beta_{V}(w) \mathrm{d} w} V_{T} I_{A_{1}, B_{2}}\right)\right], \\
& P_{0}^{d}=E\left[\left(e^{-r T} X-e^{-\int_{0}^{T} \beta_{S}(w) \mathrm{d} w} S_{T}\right)\left(I_{A_{2}, B_{1}}+\frac{1-\alpha}{e^{-r T} D} e^{-\int_{0}^{T} \beta_{V}(w) \mathrm{d} w} V_{T} I_{A_{2}, B_{2}}\right)\right],
\end{aligned}
$$

where $I_{(.)}$is an indicator function. $A_{i}$ and $B_{i}(i=1,2)$ are given by

$$
\begin{aligned}
& A_{1}=e^{-\int_{0}^{T} \beta_{S}(w) \mathrm{d} w} S_{T} \geq e^{-r T} X, \\
& A_{2}=e^{-\int_{0}^{T} \beta_{S}(w) \mathrm{d} w} S_{T}<e^{-r T} X, \\
& B_{1}=e^{-\int_{0}^{T} \beta_{V}(w) \mathrm{d} w} V_{T} \geq e^{-r T} D^{*}, \\
& B_{2}=e^{-\int_{0}^{T} \beta_{V}(w) \mathrm{d} w} V_{T}<e^{-r T} D^{*} .
\end{aligned}
$$

$A_{1}$ and $A_{2}$ are the essential conditions for performing the European vulnerable call and put option on the maturity date $T$, respectively. $B_{1}$ and $B_{2}$ indicate whether a credit loss occurs or not.

\section{Pricing for Vulnerable Options in the Jump BFBM Environment}

In this section, we derived closed-form pricing formulae for European vulnerable options in the BFBM environment with jumps by actuarial approach. Taking the long-range dependence and jumps in the underlying asset price and the firm value of the counterparty into consideration, we adopt jump bifractional Brownian motions to describe the dynamics of the underlying asset price and the firm value of the counterparty. As in [18], we divide the jump term in the underlying asset price into the individual jumps and the common jumps as well as the firm value of the counterparty. 
Now, our pricing model is described in detail as follows.

\subsection{Financial Model Description}

Assumption 1. Let $S_{t}$ be the underlying asset price. $S_{t}$ is given by the following jump-diffusion process:

$$
\mathrm{d} S_{t}=S_{t}\left[\left(\mu_{S}(t)-\lambda_{S}^{*} \theta_{S}\right) \mathrm{d} t+\sigma_{S} \mathrm{~d} B_{S}^{H, K}(t)+\left(J_{S}(t)-1\right) \mathrm{d} M_{S}(t)\right]
$$

where $\mu_{S}(t)$, which is a determinate function of time $t$, is the instantaneous expected return of $S_{t}, \sigma_{S}$ is its volatility, $B_{S}^{H, K}(t)$ is the BFBM, and $J_{S}(t)-1$ is the percentage jump of $S_{t}$ with mean $E\left(J_{S}(t)-1\right)=\theta_{S}$. Specially, for different time, $J_{S}(t)$ is a sequence of lognormal, independent, and identical distributed with mean $\mu_{1}$ and variance $\sigma_{1}^{2}$ by Merton [3]. Thus, $\theta_{S}=e^{\mu_{1}+\left(\sigma_{1}^{2} / 2\right)}-1$. The jump term $\left(J_{S}(t)-1\right) \mathrm{d} M_{S}(t)$ describes discontinuous changes in the underlying asset price. $M_{S}(t)$ denotes a Poisson process with intensity $\lambda_{S}^{*}$. In our framework, jump term $M_{S}(t)$ and the jump intensity $\lambda_{S}^{*}$ are both composed of two parts:

$$
\begin{aligned}
M_{S}(t) & =N_{S}(t)+N(t), \\
\lambda_{S}^{*} & =\lambda_{S}+\lambda .
\end{aligned}
$$

Moreover, sudden shocks occurred in the underlying asset price consist of two parts. One part is individual shocks which correspond to $N_{S}(t)$. The other part is common shocks linked to market factors, corresponding to $N(t)$ which affects the prices of the underlying asset and the firm value of the counterparty, where $N_{S}(t)$ with the intensity $\lambda_{S}$ and $N(t)$ with the intensity $\lambda$ are independent Poisson processes.

Assumption 2. Let $V_{t}$ be the firm value of the counterparty. The dynamics of $V_{t}$ is governed by the following jumpdiffusion process:

$$
\mathrm{d} V_{t}=V_{t}\left[\left(\mu_{V}(t)-\lambda_{V}^{*} \theta_{V}\right) \mathrm{d} t+\sigma_{V} \mathrm{~d} B_{V}^{H, K}(t)+\left(J_{V}(t)-1\right) \mathrm{d} M_{V}(t)\right]
$$

where $\mu_{V}(t), \lambda_{V}^{*}, \theta_{V}, \sigma_{V}$, and $B_{V}^{H, K}(t)$ are the expected return of $V_{t}$, the jump intensity of the Poisson process $M_{V}(t)$, the mean percentage jump of $V_{t}$, the volatility of $V_{t}$, and the BFBM, respectively. $J_{V}(t)-1$ is the percentage jump. For different time, $J_{V}(t)$ is a sequence of lognormal, independent, and identical distributed with mean $\mu_{2}$ and variance $\sigma_{2}^{2}$ as in [3]. Then, $\theta_{V}=e^{\mu_{2}+\left(\sigma_{2}^{2} / 2\right)}-1$. Similarly, $M_{V}(t)$ and $\lambda_{V}^{*}$ are both expressed as

$$
\begin{aligned}
M_{V}(t) & =N_{V}(t)+N(t), \\
\lambda_{V}^{*} & =\lambda_{V}+\lambda,
\end{aligned}
$$

where $N_{V}(t)$, which denotes individual jumps, is a Poisson process with intensity $\lambda_{V}$ and independent of $N(t)$ and $N_{S}(t)$.

Further, it is supposed that $B_{S}^{H, K}(t)$ and $B_{V}^{H, K}(t)$ are two correlated BFBM with correlation coefficient $\rho_{S V}$, these processes $N(t), N_{S}(t), N_{V}(t), \ln J_{S}(t), \ln J_{V}(t), B_{i}^{H, K}(t)$, $(i \in S, V)$ are mutually independent.

3.2. Pricing Formulae for Vulnerable Options. In order to derive the closed-form pricing formulae for European vulnerable options, we need the following lemma.

Lemma 1. The expectation yield rates $\beta_{S}(w)$ and $\beta_{V}(w)$, given by (6) and (7), respectively, satisfy the following equalities:

$$
\begin{aligned}
& \int_{0}^{T} \beta_{S}(w) \mathrm{d} w=\int_{0}^{T} \mu_{S}(w) \mathrm{d} w \\
& \int_{0}^{T} \beta_{V}(w) \mathrm{d} w=\int_{0}^{T} \mu_{V}(w) \mathrm{d} w .
\end{aligned}
$$

Proof. By applying bifractional Itô formula by Es-Sebaiy and Tudor [49], it is easy to obtain the solution of equation (11):

$$
S_{T}=S_{0} \mathrm{e}^{\int_{0}^{T} \mu_{S}(w) \mathrm{d} w-\lambda_{S}^{*} \theta_{S} T-(1 / 2) \sigma_{S}^{2} T^{2 H K}+\sigma_{S} B_{S}^{H, K}(T)+\sum_{i=1}^{M_{S}(T)} \ln J_{S}\left(t_{i}\right)}
$$

where $t_{i}$ denotes the $i$ th jump time of $M_{S}(T)$. For different time, $\ln J_{S}\left(t_{i}\right)$ is independent normally distributed with mean $\mu_{1}$ and variance $\sigma_{1}^{2}$, as in [3].

Noting that $B_{S}^{H, K}(T) \sim N\left(0, T^{2 H K}\right), E\left[e^{\sigma_{S} B_{S}^{H, K}(T)}\right]$ $=e^{(1 / 2) \sigma_{S}^{2} T^{2 H K}}$, the mutual independence of $B_{S}^{H, K}(T), M_{S}(T)$ and $\ln J_{S}\left(t_{i}\right)$, and the theory of Poisson distribution with intensity $\lambda_{S}^{*} T$, then we can get

$$
\frac{E\left(S_{T}\right)}{S_{0}}=e^{\int_{0}^{T} \mu_{S}(w) \mathrm{d} w-\lambda_{S}^{*} \theta_{S} T-(1 / 2) \sigma_{S}^{2} T^{2 H K}} E\left(\exp \left(B_{S}^{H, K}(T)\right) E\left[\exp \left(\sum_{i=1}^{M_{S}(T)} \ln J_{S}\left(t_{i}\right)\right)\right]\right.
$$




$$
\begin{aligned}
& =e^{\int_{0}^{T} \mu_{S}(w) \mathrm{d} w-\lambda_{S}^{*} \theta_{S} T} E\left[\exp \left(\sum_{i=1}^{M_{S}(T)} \ln J_{S}\left(t_{i}\right)\right)\right] \\
& =e^{\int_{0}^{T} \mu_{S}(w) \mathrm{d} w-\lambda_{S}^{*} \theta_{S} T} E\left[E\left[\exp \left(\sum_{i=1}^{M_{S}(T)} \ln J_{S}\left(t_{i}\right)\right)\right] \mid M_{S}(T)\right] \\
& =e^{\int_{0}^{T} \mu_{S}(w) \mathrm{d} w-\lambda_{S}^{*} \theta_{S} T} \sum_{m=0}^{\infty} P\left(M_{S}(T)=m\right) E\left[\exp \left(\sum_{i=0}^{m} \ln J_{S}\left(t_{i}\right)\right) \mid M_{S}(T)=m\right] \\
& =e^{\int_{0}^{T} \mu_{s}(w) \mathrm{d} w-\lambda_{S}^{*} \theta_{S} T} \sum_{m=0}^{\infty} P\left(M_{S}(T)=m\right)\left(E\left(J_{S}\left(t_{i}\right)\right)\right)^{m} \\
& =e^{\int_{0}^{T} \mu_{s}(w) \mathrm{d} w-\lambda_{S}^{*}\left(1+\theta_{S}\right) T} \sum_{m=0}^{\infty} \frac{\left(\lambda_{S}^{*} T\right)^{m}}{m !}\left(1+\theta_{S}\right)^{m} \\
& =e^{\int_{0}^{T} \mu_{s}(w) \mathrm{d} w} .
\end{aligned}
$$

Combing (6) and (18), the proof of (15) is completed. Similarly, we can also show that the equality (16) is satisfied.
Theorem 1. Assume that the underlying asset price $S_{t}$ and the firm value of the counterparty $V_{t}$ are given by (11) and (12). Thus, the explicit formulae for European vulnerable option prices are expressed as

$$
\begin{aligned}
C_{0}^{d}= & \sum_{n=0}^{\infty} \sum_{m_{1}=n}^{\infty} \sum_{m_{2}=n}^{\infty} P_{n}(\lambda T) P_{m_{1}-n}\left(\lambda_{S} T\right) P_{m_{2}-n}\left(\lambda_{V} T\right) \\
& \cdot\left(S_{0} e^{-\lambda_{S}^{*} \theta_{S} T+m_{1}\left(\mu_{1}+\left(\sigma_{1}^{2} / 2\right)\right)} N_{2}\left(a_{1}\left(m_{1}\right), a_{2}\left(m_{2}\right), \rho\right)-X e^{-r T} N_{2}\left(b_{1}\left(m_{1}\right), b_{2}\left(m_{2}\right), \rho\right)\right. \\
& +\frac{1-\alpha}{D} S_{0} V_{0} \mathrm{e}^{\left(r-\lambda_{S}^{*} \theta_{S}-\lambda_{V}^{*} \theta_{V}\right) T+m_{1}\left(\mu_{1}+\left(\sigma_{1}^{2} / 2\right)\right)+m_{2}\left(\mu_{2}+\left(\sigma_{2}^{2} / 2\right)\right)+\rho \delta_{S} \delta_{V}} N_{2}\left(c_{1}\left(m_{1}\right),-c_{2}\left(m_{2}\right),-\rho\right) \\
& \left.-\frac{1-\alpha}{D} X V_{0} \mathrm{e}^{-\lambda_{V}^{*} \theta_{V} T+m_{2}\left(\mu_{2}+\left(\sigma_{2}^{2} / 2\right)\right)} N_{2}\left(d_{1}\left(m_{1}\right),-d_{2}\left(m_{2}\right),-\rho\right)\right), \\
P_{0}^{d}= & \sum_{n=0}^{\infty} \sum_{m_{1}=n}^{\infty} \sum_{m_{2}=n}^{\infty} P_{n}(\lambda T) P_{m_{1}-n}\left(\lambda_{S} T\right) P_{m_{2}-n}\left(\lambda_{V} T\right) \\
& \cdot\left(X e^{-r T} N_{2}\left(-b_{1}\left(m_{1}\right), b_{2}\left(m_{2}\right),-\rho\right)-S_{0} e^{-\lambda_{S}^{*} \theta_{S} T+m_{1}\left(\mu_{1}+\left(\sigma_{1}^{2} / 2\right)\right)} N_{2}\left(-a_{1}\left(m_{1}\right), a_{2}\left(m_{2}\right),-\rho\right)\right. \\
& +\frac{1-\alpha}{D} X V_{0} \mathrm{e}^{-\lambda_{V}^{*} \theta_{V} T+m_{2}\left(\mu_{2}+\left(\sigma_{2}^{2} / 2\right)\right)} N_{2}\left(-d_{1}\left(m_{1}\right),-d_{2}\left(m_{2}\right), \rho\right) \\
& \left.-\frac{1-\alpha}{D} S_{0} V_{0} \mathrm{e}^{\left(r-\lambda_{S}^{*} \theta_{S}-\lambda_{V}^{*} \theta_{V}\right) T+m_{1}\left(\mu_{1}+\left(\sigma_{1}^{2} / 2\right)\right)+m_{2}\left(\mu_{2}+\left(\sigma_{2}^{2} / 2\right)\right)+\rho \delta_{S} \delta_{V}} N_{2}\left(-c_{1}\left(m_{1}\right),-c_{2}\left(m_{2}\right), \rho\right)\right),
\end{aligned}
$$

where $P_{n}(\lambda T), P_{m_{1}-n}\left(\lambda_{S} T\right)$, and $P_{m_{2}-n}\left(\lambda_{V} T\right)$ are the probability functions of $N(T), N_{S}(T)$, and $N_{V}(T)$ throughout the paper, respectively, with $P_{n}(\lambda T)=\left((\lambda T)^{n} / n !\right) e^{-\lambda T}, P_{m_{1}-n}$
$\left(\lambda_{S} T\right)=\left(\left(\lambda_{S} T\right)^{m_{1}-n} /\left(m_{1}-n\right) !\right) e^{-\lambda_{S} T}, P_{m_{2}-n}\left(\lambda_{V} T\right)=\left(\left(\lambda_{V} T\right.\right.$ )$\left.^{m_{2}-n} /\left(m_{2}-n\right) !\right) e^{-\lambda_{V} T} \cdot N_{2}(\cdot)$ denotes the bivariate normal cumulative distribution function given by 


$$
\begin{aligned}
N_{2}(x, y, \rho) & =\frac{1}{2 \pi \sqrt{1-\rho^{2}}} \int_{-\infty}^{x} \int_{-\infty}^{y} e^{-\left(1 / 2\left(1-\rho^{2}\right)\right)\left(u^{2}-2 \rho u v+v^{2}\right)} \mathrm{d} u \mathrm{~d} v \\
a_{1}\left(m_{1}\right) & =b_{1}\left(m_{1}\right)+\delta_{S} \\
a_{2}\left(m_{2}\right) & =b_{2}\left(m_{2}\right)+\rho \delta_{S} \\
b_{1}\left(m_{1}\right) & =\frac{\ln \left(S_{0} / X\right)+\left(r-\lambda_{S}^{*} \theta_{S}\right) T+m_{1} \mu_{1}-\left(\sigma_{S}^{2} T^{2 H K} / 2\right)}{\delta_{S}} \\
b_{2}\left(m_{2}\right) & =\frac{\ln \left(V_{0} / D^{*}\right)+\left(r-\lambda_{V}^{*} \theta_{V}\right) T+m_{2} \mu_{2}-\left(\sigma_{V}^{2} T^{2 H K} / 2\right)}{\delta_{V}} \\
c_{1}\left(m_{1}\right) & =b_{1}\left(m_{1}\right)+\delta_{S}+\rho \delta_{V}, \\
c_{2}\left(m_{2}\right) & =b_{2}\left(m_{2}\right)+\delta_{V}+\rho \delta_{S} \\
d_{1}\left(m_{1}\right) & =b_{1}\left(m_{1}\right)+\rho \delta_{V}, \\
d_{2}\left(m_{2}\right) & =b_{2}\left(m_{2}\right)+\delta_{V}, \\
\delta_{S}^{2} & =\sigma_{S}^{2} T^{2 H K}+m_{1} \sigma_{1}^{2}, \\
\delta_{V}^{2} & =\sigma_{V}^{2} T^{2 H K}+m_{2} \sigma_{2}^{2}, \rho=\frac{\rho_{S V} \sigma_{S} \sigma_{V} T^{2 H K}}{\delta_{S} \delta_{V}} .
\end{aligned}
$$

Proof. We only show the pricing formula (19) for a European vulnerable call option which is satisfied in this part. The corresponding put option price (20) can also be derived by the similar calculations.

For the sake of simplicity, (8) can be rewritten as

$$
C_{0}^{d}=I_{1}-I_{2}+I_{3}-I_{4}
$$

where $I_{1}, I_{2}, I_{3}, I_{4}$ are given by

$$
\begin{aligned}
& I_{1}=E\left[e^{-\int_{0}^{T} \beta_{S}(w) \mathrm{d} w} S_{T} I_{A_{1}, B_{1}}\right], \\
& I_{2}=X e^{-r T} E\left[I_{A_{1}, B_{1}}\right],
\end{aligned}
$$

$$
\begin{aligned}
& I_{3}=\frac{1-\alpha}{e^{-r T} D} E\left[e^{-\int_{0}^{T} \beta_{S}(w) \mathrm{d} w} S_{T} e^{-\int_{0}^{T} \beta_{V}(w) \mathrm{d} w} V_{T} I_{A_{1}, B_{2}}\right], \\
& I_{4}=\frac{(1-\alpha) X}{D} E\left[e^{-\int_{0}^{T} \beta_{V}(w) \mathrm{d} w} V_{T} I_{A_{1}, B_{2}}\right] .
\end{aligned}
$$

In order to calculate $I_{1}, I_{2}, I_{3}, I_{4}$, we give some notations:

$$
\begin{aligned}
S_{T}^{m_{1}} & =S_{0} \mathrm{e}_{0}^{T} \mu_{S}(w) \mathrm{d} w-\lambda_{S}^{*} \theta_{S} T-(1 / 2) \sigma_{S}^{2} T^{2 H K}+\sigma_{S} B_{S}^{H, K}(T)+\sum_{i=1}^{m_{1}} \ln J_{S}\left(t_{i}\right), \\
V_{T}^{m_{2}} & =V_{0} \mathrm{e}_{0}^{T} \mu_{V}(w) \mathrm{d} w-\lambda_{V}^{*} \theta_{V} T-(1 / 2) \sigma_{V}^{2} T^{2 H K}+\sigma_{V} B_{V}^{H, K}(T)+\sum_{i=1}^{m_{2}} \ln J_{V}\left(t_{i}\right), \\
z_{1} & =\frac{\sigma_{S} B_{S}^{H, K}(T)+\sum_{i=1}^{m_{1}} \ln J_{S}\left(t_{i}\right)-m_{1} \mu_{1}}{\delta_{S}}, \\
\delta_{S}^{2} & =\left(\sigma_{S}\right)^{2} T^{2 H K}+m_{1} \sigma_{1}^{2},
\end{aligned}
$$




$$
\begin{aligned}
z_{2} & =\frac{\sigma_{V} B_{V}^{H, K}(T)+\sum_{i=1}^{m_{2}} \ln J_{V}\left(t_{i}\right)-m_{2} \mu_{2}}{\delta_{V}}, \\
\delta_{V}^{2} & =\left(\sigma_{V}\right)^{2} T^{2 H K}+m_{2} \sigma_{2}^{2}, \\
b_{1}\left(m_{1}\right) & =\frac{\ln \left(S_{0} / X\right)+\left(r-\lambda_{S} \theta_{S}\right) T+m_{1} \mu_{1}-\left(\sigma_{S}^{2} T^{2 H K} / 2\right)}{\delta_{S}}, \\
b_{2}\left(m_{2}\right) & =\frac{\ln \left(V_{0} / D^{*}\right)+\left(r-\lambda_{V} \theta_{V}\right) T+m_{2} \mu_{2}-\left(\sigma_{V}^{2} T^{2 H K} / 2\right)}{\delta_{V}} .
\end{aligned}
$$

Now, we deal with each of these terms $I_{1}, I_{2}, I_{3}, I_{4}$ separately.

Suppose that conditional on $\mathscr{G}_{T}^{n, n_{1}, n_{2}}:=\{N(T)=$ $\left.n, N_{S}(T)=n_{1}, N_{V}(T)=n_{2}\right\}$, the total jump times of $S_{T}$ and $V_{T}$ are denoted by $m_{1} \triangleq n+n_{1}, m_{2} \triangleq n+n_{2}$, for simplicity.
Applying the mutual independence of $N(t), N_{S}(t)$, $N_{V}(t), \ln J_{S}(t), \ln J_{V}(t), B_{i}^{H, K}(t)(i \in S, V)$, equations (11) and (13) and Lemma 1, we have

$$
\begin{aligned}
& I_{2}=X e^{-r T} E\left(I_{A_{1}, B_{1}}\right) \\
& =X e^{-r T} E\left[E\left(I_{A_{1}, B_{1}} \mid N(T), N_{S}(T), N_{V}(T)\right)\right] \\
& =X e^{-r T} \sum_{n=0}^{\infty} \sum_{n_{1}=0}^{\infty} \sum_{n_{2}=0}^{\infty} P_{n}(\lambda T) P_{n_{1}}\left(\lambda_{S} T\right) P_{n_{2}}\left(\lambda_{V} T\right) \\
& \cdot E\left[I I_{e^{-\int_{0}^{T} \beta_{S}(w) \mathrm{d} w} S_{T}^{m_{1}} \geq e^{-r T} K, e^{-\int_{0}^{T} \beta_{V}(w) \mathrm{d} w} V_{T}^{m_{2}} \geq e^{-r T} D^{*}} \mid N(T)=n, N_{S}(T)=n_{1}, N_{V}(T)=n_{2}\right] \\
& =X e^{-r T} \sum_{n=0}^{\infty} \sum_{m_{1}=n}^{\infty} \sum_{m_{2}=n}^{\infty} P_{n}(\lambda T) P_{m_{1}-n}\left(\lambda_{S} T\right) P_{m_{2}-n}\left(\lambda_{V} T\right) E\left(I_{z_{1} \geq-b_{1}\left(m_{1}\right), z_{2} \geq-b_{2}\left(m_{2}\right)}\right) \text {. }
\end{aligned}
$$

Noting that $B_{S}^{H, K}(T) \sim N\left(0, T^{2 H K}\right), B_{V}^{H, K}(T) \sim$ $N\left(0, T^{2 H K}\right), \operatorname{cov}\left(B_{S}^{H, K}(T), B_{V}^{H, K}(T)\right)=\rho_{S V} T^{2 H K}, \ln J_{S}\left(t_{i}\right)$ is independent normally distributed with mean $\mu_{1}$ and variance $\sigma_{1}^{2}$, then $\left(z_{1}, z_{2}\right)$ obey standard bivariate normal distribution with the correlation $\rho=\left(\rho_{S V} \sigma_{S} \sigma_{V} T^{2 H K} / \delta_{S} \delta_{V}\right)$. Therefore, we obtain

$$
\begin{aligned}
I_{2}= & X e^{-r T} \sum_{n=0}^{\infty} \sum_{m_{1}=n}^{\infty} \sum_{m_{2}=n}^{\infty} P_{n}(\lambda T) P_{m_{1}-n}\left(\lambda_{S} T\right) P_{m_{2}-n} \\
& \left(\lambda_{V} T\right) N_{2}\left(b_{1}\left(m_{1}\right), b_{2}\left(m_{2}\right), \rho\right) .
\end{aligned}
$$

Similar to the calculation of $I_{2}$, we have

$$
\begin{aligned}
I_{1}= & E\left[E\left(e^{-\int_{0}^{T} \beta_{S}(w) \mathrm{d} w} S_{T} I_{A_{1}, B_{1}} \mid N(T), N_{S}(T), N_{V}(T)\right)\right] \\
= & \sum_{n=0}^{\infty} \sum_{n_{1}=0}^{\infty} \sum_{n_{2}=0}^{\infty} P_{n}(\lambda T) P_{n_{1}}\left(\lambda_{S} T\right) P_{n_{2}}\left(\lambda_{V} T\right) S_{0} e^{-\lambda_{S}^{*} \theta_{S} T+m_{1}\left(\mu_{1}+\left(\sigma_{1}^{2} / 2\right)\right)} \\
& \cdot E\left[e^{\left.\delta_{S} z_{1}-\left(\delta^{2} s / 2\right) I_{z_{1} \geq-b_{1}\left(m_{1}\right), z_{2} \geq-b_{2}\left(m_{2}\right)} \mid N(T)=n, N_{S}(T)=n_{1}, N_{V}(T)=n_{2}\right]}\right. \\
= & \sum_{n=0}^{\infty} \sum_{m_{1}=n}^{\infty} \sum_{m_{2}=n}^{\infty} P_{n}(\lambda T) P_{m_{1}-n}\left(\lambda_{S} T\right) P_{m_{2}-n}\left(\lambda_{V} T\right) S_{0} \mathrm{e}^{-\lambda_{S}^{*} \theta_{S} T+m_{1}\left(\mu_{1}+\left(\sigma_{1}^{2} / 2\right)\right)} \\
& \cdot \int_{-b_{1}\left(m_{1}\right)}^{+\infty} \int_{-b_{2}\left(m_{2}\right)}^{+\infty} e^{\delta_{S} z_{1}-\left(\delta^{2} s / 2\right)} \varphi\left(z_{1}, z_{2}, \rho\right) \mathrm{d} z_{1} \mathrm{~d} z_{2},
\end{aligned}
$$


where $\varphi\left(z_{1}, z_{2}, \rho\right)$, the standard bivariate normal probability density function, is given by

$$
\varphi\left(z_{1}, z_{2}, \rho\right)=\frac{1}{2 \pi \sqrt{1-\rho^{2}}} e^{-\left(1 / 2\left(1-\rho^{2}\right)\right)\left(z_{1}^{2}+z_{2}^{2}-2 \rho z_{1} z_{2}\right)} .
$$

Applying the method of undetermined coefficients to equation (27), the exponent of the integrand in (27) is of the following form:

$$
-\frac{1}{2\left(1-\rho^{2}\right)}\left(\left(z_{1}+a\right)^{2}+\left(z_{2}+b\right)^{2}-2 \rho\left(z_{1}+a\right)\left(z_{2}+b\right)\right)+M
$$

where $a=-\delta_{S}, b=-\rho \delta_{S}, M=0$. Setting $a_{1}\left(m_{1}\right)=b_{1}\left(m_{1}\right)$ $+\delta_{S}, a_{2}\left(m_{2}\right)=b_{2}\left(m_{2}\right)+\rho \delta_{S}$, then we obtain

$$
\begin{aligned}
& I_{1}=\sum_{n=0}^{\infty} \sum_{m_{1}=n}^{\infty} \sum_{m_{2}=n}^{\infty} P_{n}(\lambda T) P_{m_{1}-n}\left(\lambda_{S} T\right) P_{m_{2}-n}\left(\lambda_{V} T\right) S_{0} e^{-\lambda_{S}^{*} \theta_{S} T+m_{1}\left(\mu_{1}+\left(\sigma_{1}^{2} / 2\right)\right)} \\
& \cdot \int_{-b_{1}\left(m_{1}\right)}^{+\infty} \int_{-b_{2}\left(m_{2}\right)}^{+\infty} \varphi\left(z_{1}-\delta_{S}, z_{2}-\rho \delta_{S}, \rho\right) \mathrm{d} z_{1} \mathrm{~d} z_{2}
\end{aligned}
$$

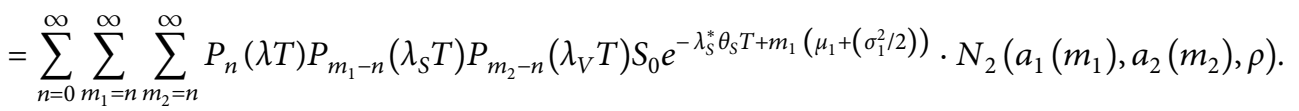

In order to calculate $I_{3}$, we observe that $e^{-\int_{0}^{T} \beta_{V}(w) \mathrm{d} w}$ $V_{T}^{m_{2}}<e^{-r T} D^{*}$ is equivalent to $z_{2}<-b_{2}\left(m_{2}\right)$, then we deduce

$$
\begin{aligned}
I_{3}= & \sum_{n=0}^{\infty} \sum_{m_{1}=n}^{\infty} \sum_{m_{2}=n}^{\infty} P_{n}(\lambda T) P_{m_{1}-n}\left(\lambda_{S} T\right) P_{m_{2}-n}\left(\lambda_{V} T\right) \frac{1-\alpha}{D} S_{0} V_{0} e^{\left(r-\lambda_{S}^{*} \theta_{S}-\lambda_{V}^{*} \theta_{V}\right) T} \\
& \cdot e^{m_{1}\left(\mu_{1}+\left(\sigma_{1}^{2} / 2\right)\right)+m_{2}\left(\mu_{2}+\left(\sigma_{2}^{2} / 2\right)\right)} \int_{-b_{1}\left(m_{1}\right)}^{+\infty} \int_{-b_{2}\left(m_{2}\right)}^{-\infty} e^{\delta_{S} z_{1}+\delta_{V} z_{2}-\left(\delta^{2} S / 2\right)} \varphi\left(z_{1}, z_{2}, \rho\right) \mathrm{d} z_{1} \mathrm{~d} z_{2} \\
= & \sum_{n=0}^{\infty} \sum_{m_{1}=n}^{\infty} \sum_{m_{2}=n}^{\infty} P_{n}(\lambda T) P_{m_{1}-n}\left(\lambda_{S} T\right) P_{m_{2}-n}\left(\lambda_{V} T\right) \frac{1-\alpha}{D} S_{0} V_{0} e^{\left(r-\lambda_{S}^{*} \theta_{S}-\lambda_{V}^{*} \theta_{V}\right) T} \\
& \cdot e^{m_{1}\left(\mu_{1}+\left(\sigma_{1}^{2} / 2\right)\right)+m_{2}\left(\mu_{2}+\left(\sigma_{2}^{2} / 2\right)\right)+\rho \delta_{S} \delta_{V}} \int_{-b_{1}\left(m_{1}\right)}^{+\infty} \int_{-\infty}^{-b_{2}\left(m_{2}\right)} \varphi\left(z_{1}-\delta_{S}-\rho \delta_{V}, z_{2}-\delta_{V}-\rho \delta_{S}, \rho\right) \mathrm{d} z_{1} \mathrm{~d} z_{2} .
\end{aligned}
$$

Setting $\quad c_{1}\left(m_{1}\right)=b_{1}\left(m_{1}\right)+\delta_{S}+\rho \delta_{V} \quad$ and $\quad c_{2}\left(m_{2}\right)=$ $b_{2}\left(m_{2}\right)+\delta_{V}+\rho \delta_{S}$, then

$$
\begin{aligned}
I_{3}= & \sum_{n=0}^{\infty} \sum_{m_{1}=n}^{\infty} \sum_{m_{2}=n}^{\infty} P_{n}(\lambda T) P_{m_{1}-n}\left(\lambda_{S} T\right) P_{m_{2}-n} \\
& \left(\lambda_{V} T\right) \frac{1-\alpha}{D} S_{0} V_{0} \mathrm{e}^{\left(r-\lambda_{S}^{*} \theta_{S}-\lambda_{V}^{*} \theta_{V}\right) T} \\
& \cdot e^{m_{1}\left(\mu_{1}+\left(\sigma_{1}^{2} / 2\right)\right)+m_{2}\left(\mu_{2}+\left(\sigma_{2}^{2} / 2\right)\right)+\rho \delta_{S} \delta_{V}} N\left(c_{1}\left(m_{1}\right),-c_{2}\left(m_{2}\right),-\rho\right) .
\end{aligned}
$$

For the term $I_{4}$. it is easy to obtain

$$
\begin{aligned}
I_{4}= & \sum_{n=0}^{\infty} \sum_{m_{1}=n}^{\infty} \sum_{m_{2}=n}^{\infty} P_{n}(\lambda T) P_{m_{1}-n}\left(\lambda_{S} T\right) P_{m_{2}-n}\left(\lambda_{V} T\right) \frac{(1-\alpha) X V_{0}}{D} \\
& \cdot e^{-\lambda_{V}^{*} \theta_{V} T+m_{2}\left(\mu_{2}+\left(\sigma_{2}^{2} / 2\right)\right)} \int_{-b_{1}\left(m_{1}\right)}^{+\infty} \int_{-\infty}^{-b_{2}\left(m_{2}\right)} \varphi \\
& \left(z_{1}-\rho \delta_{V}, z_{2}-\delta_{V}, \rho\right) \mathrm{d} z_{1} \mathrm{~d} z_{2} .
\end{aligned}
$$


Setting $d_{1}\left(m_{1}\right)=b_{1}\left(m_{1}\right)+\rho \delta_{V}$ and $d_{2}\left(m_{2}\right)=b_{2}\left(m_{2}\right)$ $+\delta_{V}$, then

$$
\begin{aligned}
I_{4}= & \sum_{n=0}^{\infty} \sum_{m_{1}=n}^{\infty} \sum_{m_{2}=n}^{\infty} P_{n}(\lambda T) P_{m_{1}-n}\left(\lambda_{S} T\right) P_{m_{2}-n}\left(\lambda_{V} T\right) \frac{(1-\alpha) X V_{0}}{D} \\
& \cdot e^{-\lambda_{V}^{*} \theta_{V} T+m_{2}\left(\mu_{2}+\left(\sigma_{2}^{2} / 2\right)\right)} N\left(d_{1}(m),-d_{2}(n),-\rho\right) .
\end{aligned}
$$

Combining the calculated $I_{1}, I_{2}, I_{3}, I_{4}$, the pricing formula (19) is derived.

3.3. Special Cases of the Pricing Formulae. Compared with many existing models such as Black-Scholes [1], Merton [3], Klein [15], and Tian et al. [18], our proposed framework not only adds jump risk and credit risk but also adds the longrange dependence of financial asset prices. Therefore, our model encompasses these existing models as special cases. The details are as follows.

Case 1. Black-Scholes model [1].

If there is no jump risk, credit risk, and long-range dependence in our model, i.e., $\lambda=\lambda_{S}=\lambda_{V}=0, n=n_{1}=n_{2}=$ $0, D^{*} \longrightarrow 0, H=(1 / 2)$ and $K=1$, our model will reduce to the Black-Scholes model. The formulae for European options under the Black-Scholes model are given by

$$
\begin{aligned}
& C_{0}=S_{0} N(a)-X e^{-r T} N(b), \\
& P_{0}=X e^{-r T} N(-b)-S_{0} N(-a),
\end{aligned}
$$

where $\quad a=b+\sigma_{S} \sqrt{T}, b=\left(\ln \left(S_{0} / X\right)+\left(r-\left(\sigma_{S}^{2} / 2\right)\right) \quad T / \sigma_{S}\right.$ $\sqrt{T}), N(\cdot)$ denotes the standard normal cumulative distribution function.

It is easily seen that $a_{1}(0)$ and $b_{1}(0)$ reduce to $a=b+$ $\sigma_{S} \sqrt{T}$ and $b=\left(\ln \left(S_{0} / X\right)+\left(r-\left(\sigma_{S}^{2} / 2\right)\right) T / \sigma_{S} \sqrt{T}\right)$. Moreover, $\quad a_{2}(0) \longrightarrow+\infty, b_{2}(0) \longrightarrow+\infty, c_{2}(0) \longrightarrow$ $+\infty, d_{2}(0) \longrightarrow+\infty$ as $D^{*} \longrightarrow 0$. Note that $N_{2}(z,-\infty, \rho) \longrightarrow 0, N_{2}(z,+\infty, \rho) \longrightarrow N(z)$, then formulae (19) and (20) can be simplified to formulae (35) and (36), respectively.

Case 2. Merton jump-diffusion model [3].

If there is no credit risk, i.e., $D^{*} \longrightarrow 0$, and the underlying asset prices are governed by geometric Brownian motion with jumps, that is, $H=(1 / 2)$ and $K=1$, then formula (19) can be rewritten as the following form:

$$
\begin{aligned}
& C_{0}^{d}=\sum_{n=0}^{\infty} \sum_{m_{1}=n}^{\infty} \sum_{m_{2}=n}^{\infty} \frac{(\lambda T)^{n}}{n !} \frac{\left(\lambda_{S} T\right)^{m_{1}-n}}{\left(m_{1}-n\right) !} \frac{\left(\lambda_{V} T\right)^{m_{2}-n}}{\left(m_{2}-n\right) !} e^{\left(-\lambda-\lambda_{S}-\lambda_{V}\right) T} \\
& \cdot\left(S_{0} e^{-\lambda_{S}^{*} \theta_{S} T+m_{1}\left(\mu_{1}+\left(\sigma_{1}^{2} / 2\right)\right)} N_{2}\left(a_{1}\left(m_{1}\right)\right)-X e^{-r T} N_{2}\left(b_{1}\left(m_{1}\right)\right)\right. \\
& =\sum_{n=0}^{\infty} \sum_{m_{1}=n}^{\infty} \frac{(\lambda T)^{n}}{n !} \frac{\left(\lambda_{S} T\right)^{m_{1}-n}}{\left(m_{1}-n\right) !} e^{\left(-\lambda-\lambda_{S}\right) T} \\
& \cdot\left(S_{0} e^{-\lambda_{S}^{*} \theta_{S} T+m_{1}\left(\mu_{1}+\left(\sigma_{1}^{2} / 2\right)\right)} N_{2}\left(a_{1}\left(m_{1}\right)\right)-X e^{-r T} N_{2}\left(b_{1}\left(m_{1}\right)\right)\right. \\
& =\sum_{i=0}^{\infty} \frac{\left(\lambda_{S}^{*} T\right)^{i}}{i !} e^{-\lambda_{S}^{*} T}\left(S_{0} e^{-\lambda_{S}^{*} \theta_{S} T+m_{1}\left(\mu_{1}+\left(\sigma_{1}^{2} / 2\right)\right)} N_{2}\left(a_{1}(i)\right)-X e^{-r T} N_{2}\left(b_{1}(i)\right),\right.
\end{aligned}
$$

where equality (37) comes from the expression $S_{0} e^{-\lambda_{S}^{*} \theta_{S} T+m_{1}\left(\mu_{1}+\left(\sigma_{1}^{2} / 2\right)\right)} N_{2}\left(a_{1}\left(m_{1}\right)\right)-X e^{-r T} N_{2}\left(b_{1}\left(m_{1}\right)\right)$ which has no relationship with $m_{2}$, $\sum_{m_{2}=n}^{\infty}\left(\left(\lambda_{V} T\right)^{m_{2}-n} /\left(m_{2}-n\right) !\right) e^{-\lambda_{V} T}=1$, and the binomial theorem $(a+b)^{n}=\sum_{k=0}^{n} C_{n}^{k} a^{k} b^{n-k}$.
Thus, formula (37) is the valuation of European call option under the Merton jump-diffusion process. Similarly, the pricing formula for the put option is

$$
P_{0}^{d}=\sum_{i=0}^{\infty} \frac{\left(\lambda_{S}^{*} T\right)^{i}}{i !} e^{-\lambda_{S}^{*} T}\left(X e^{-r T} N_{2}\left(-b_{1}(i)-S_{0} e^{-\lambda_{S}^{*} \theta_{S} T+m_{1}\left(\mu_{1}+\left(\sigma_{1}^{2} / 2\right)\right)} N_{2}\left(-a_{1}(i)\right)\right)\right.
$$

Case 3. Klein model [15].

If no jump occurs in the underlying asset prices as well as the firm value of the counterparty, that is,
$\lambda=\lambda_{S}=\lambda_{V}, n=n_{1}=n_{2}=0$, and the underlying asset prices and the firm value of the counterparty both obey geometric Brownian motion, i.e., $H=(1 / 2)$ and $K=1$, formulae (19) 
and (20) for the European vulnerable options, which become the Klein's pricing formulae, are represented as follows:

$$
\begin{aligned}
C_{0}^{d}= & S_{0} N_{2}\left(a_{1}, a_{2}, \rho_{S V}\right)-X e^{-r T} N_{2}\left(b_{1}, b_{2}, \rho_{S V}\right) \\
& +\frac{1-\alpha}{D} S_{0} V_{0} e^{r T+\rho_{S V} \sigma_{S} \sigma_{V} T} N_{2}\left(c_{1},-c_{2},-\rho_{S V}\right)-\frac{1-\alpha}{D} X V_{0} N_{2}\left(d_{1},-d_{2},-\rho_{S V}\right), \\
P_{0}^{d}= & X e^{-r T} N_{2}\left(-b_{1}, b_{2},-\rho_{S V}\right)-S_{0} N_{2}\left(-a_{1}, a_{2},-\rho_{S V}\right) \\
& +\frac{1-\alpha}{D} X V_{0} N_{2}\left(-d_{1},-d_{2}, \rho_{S V}\right)-\frac{1-\alpha}{D} S_{0} V_{0} e^{r T+\rho_{S V} \sigma_{S} \sigma_{V} T} N_{2}\left(-c_{1},-c_{2}, \rho_{S V}\right),
\end{aligned}
$$

where

$$
\begin{aligned}
& a_{1}=b_{1}+\sigma_{S} \sqrt{T}, \\
& a_{2}=b_{2}+\rho_{S V} \sigma_{S} \sqrt{T}, \\
& b_{1}=\frac{\ln \left(S_{0} / X\right)+\left(r-\left(\sigma_{S}^{2} / 2\right)\right) T}{\sigma_{S} \sqrt{T}}, \\
& b_{2}=\frac{\ln \left(V_{0} / D^{*}\right)+\left(r-\left(\sigma_{V}^{2} / 2\right)\right) T}{\sigma_{V} \sqrt{T}}, \\
& c_{1}=b_{1}+\sigma_{S} \sqrt{T}+\rho_{S V} \sigma_{V} \sqrt{T}, \\
& c_{2}=b_{2}+\sigma_{V} \sqrt{T}+\rho_{S V} \sigma_{S} \sqrt{T}, \\
& d_{1}=b_{1}+\rho_{S V} \sigma_{V} \sqrt{T}, \\
& d_{2}=b_{2}+\sigma_{V} \sqrt{T} .
\end{aligned}
$$

Case 4. Vulnerable options under jump-diffusion processes in [18].

When $H=(1 / 2), K=1$, our jump bifractional Brownian motion model for pricing vulnerable options will reduce to the jump-diffusion model in the Brownian motion environment which was studied by Tian et al. [18]. Here, the pricing formulae (19) and (20) for European vulnerable options can be restated as follows:

$$
\begin{aligned}
C_{0}^{d}= & \sum_{n=0}^{\infty} \sum_{m_{1}=n}^{\infty} \sum_{m_{2}=n}^{\infty} P_{n}(\lambda T) P_{m_{1}-n}\left(\lambda_{S} T\right) P_{m_{2}-n}\left(\lambda_{V} T\right) \\
& \cdot\left(S_{0} e^{-\lambda_{S}^{*} \theta_{S} T+m_{1}\left(\mu_{1}+\left(\sigma_{1}^{2} / 2\right)\right)} N_{2}\left(\widehat{a_{1}}\left(m_{1}\right), \widehat{a_{2}}\left(m_{2}\right), \widehat{\rho}\right)-X e^{-r T} N_{2}\left(\widehat{b_{1}}\left(m_{1}\right), \widehat{b_{2}}\left(m_{2}\right), \widehat{\rho}\right)\right. \\
& +\frac{1-\alpha}{D} S_{0} V_{0} \mathrm{e}^{\left(r-\lambda_{S}^{*} \theta_{S}-\lambda_{V}^{*} \theta_{V}\right) T+m_{1}\left(\mu_{1}+\left(\sigma_{1}^{2} / 2\right)\right)+m_{2}\left(\mu_{2}+\left(\sigma_{2}^{2} / 2\right)\right)+\widehat{\rho} \widehat{\delta}_{S} \widehat{\delta_{V}}} N_{2}\left(\widehat{c_{1}}\left(m_{1}\right),-\widehat{c_{2}}\left(m_{2}\right),-\widehat{\rho}\right) \\
& \left.-\frac{1-\alpha}{D} X V_{0} \mathrm{e}^{-\lambda_{V}^{*} \theta_{V} T+m_{2}\left(\mu_{2}+\left(\sigma_{2}^{2} / 2\right)\right)} N_{2}\left(\widehat{d}_{1}\left(m_{1}\right),-\widehat{d_{2}}\left(m_{2}\right),-\widehat{\rho}\right)\right), \\
P_{0}^{d}= & \sum_{n=0}^{\infty} \sum_{m_{1}=n}^{\infty} \sum_{m_{2}=n}^{\infty} P_{n}(\lambda T) P_{m_{1}-n}\left(\lambda_{S} T\right) P_{m_{2}-n}\left(\lambda_{V} T\right) \\
& \cdot\left(X e^{-r T} N_{2}\left(-\widehat{b_{1}}\left(m_{1}\right), \widehat{b_{2}}\left(m_{2}\right),-\widehat{\rho}\right)-S_{0} e^{-\lambda_{S}^{*} \theta_{S} T+m_{1}\left(\mu_{1}+\left(\sigma_{1}^{2} / 2\right)\right)} N_{2}\left(-\widehat{a_{1}}\left(m_{1}\right), \widehat{a_{2}}\left(m_{2}\right),-\widehat{\rho}\right)\right. \\
& +\frac{1-\alpha}{D} X V_{0} \mathrm{e}^{-\lambda_{V}^{*} \theta_{V} T+m_{2}\left(\mu_{2}+\left(\sigma_{2}^{2} / 2\right)\right)} N_{2}\left(-\widehat{d_{1}}\left(m_{1}\right),-\widehat{d_{2}}\left(m_{2}\right), \widehat{\rho}\right) \\
& \left.-\frac{1-\alpha}{D} S_{0} V_{0} \mathrm{e}^{\left(r-\lambda_{S}^{*} \theta_{S}-\lambda_{V}^{*} \theta_{V}\right) T+m_{1}\left(\mu_{1}+\left(\sigma_{1}^{2} / 2\right)\right)+m_{2}\left(\mu_{2}+\left(\sigma_{2}^{2} / 2\right)\right)+\widehat{\rho} \widehat{\delta}_{S} \widehat{\delta_{V}}} N_{2}\left(-\widehat{c_{1}}\left(m_{1}\right),-\widehat{c_{2}}\left(m_{2}\right), \widehat{\rho}\right)\right),
\end{aligned}
$$


TABLE 1: Model comparisons.

\begin{tabular}{lcccc}
\hline Pricing models & Jump risk & Credit risk & Long-range dependence & Pricing formulae \\
\hline Black-Scholes model [1] & - & - & - & $(35),(36)$ \\
Merton model [3] & Poisson jump & - & - & $(37),(38)$ \\
Klein model [15] & - & Structural approach & - & $(39),(40)$ \\
Tian et al. pricing model [18] & Poisson jump & Structural approach & - & $(42),(43)$ \\
Our proposed model & Poisson jump & Structural approach & Bifractional Brownian motion & $(19),(20)$ \\
\hline
\end{tabular}

where

$$
\begin{aligned}
& \widehat{a_{1}}\left(m_{1}\right)=\widehat{b_{1}}\left(m_{1}\right)+\widehat{\delta_{S}}, \\
& \widehat{a_{2}}\left(m_{2}\right)=\widehat{b_{2}}\left(m_{2}\right)+\widehat{\rho} \widehat{\delta_{S}}, \\
& \widehat{b_{1}}\left(m_{1}\right)=\frac{\ln \left(S_{0} / X\right)+\left(r-\left(\sigma_{S}^{2} / 2\right)-\lambda_{S}^{*} \theta_{S}\right) T+m_{1} \mu_{1}}{\widehat{\delta_{S}}}, \\
& \widehat{b_{2}}\left(m_{2}\right)=\frac{\ln \left(V_{0} / D^{*}\right)+\left(r-\left(\sigma_{V}^{2} / 2\right)-\lambda_{V}^{*} \theta_{V}\right) T+m_{2} \mu_{2}}{\widehat{\delta_{V}}}, \\
& \widehat{c_{1}}\left(m_{1}\right)=\widehat{b_{1}}\left(m_{1}\right)+\widehat{\delta_{S}}+\widehat{\rho} \widehat{\delta_{V}}, \\
& \widehat{c_{2}}\left(m_{2}\right)=\widehat{b_{2}}\left(m_{2}\right)+\widehat{\delta_{V}}+\widehat{\rho} \widehat{\delta_{S}} \text {, } \\
& \widehat{d_{1}}\left(m_{1}\right)=\widehat{b_{1}}\left(m_{1}\right)+\widehat{\rho} \widehat{\delta_{V}}, \\
& \widehat{d}_{2}\left(m_{2}\right)=\widehat{b_{2}}\left(m_{2}\right)+\widehat{\delta_{V}}, \\
& \widehat{\delta}_{S}^{2}=\sigma_{S}^{2} T+m_{1} \sigma_{1}^{2} \\
& {\widehat{\delta_{V}}}^{2}=\sigma_{V}^{2} T^{2 H K}+m_{2} \sigma_{2}^{2}, \\
& \widehat{\rho}=\frac{\rho_{S V} \sigma_{S} \sigma_{V} T}{\widehat{\delta_{S}} \widehat{\delta_{V}}} .
\end{aligned}
$$

In conclusion, the relationship among the models mentioned above is summarized in Table 1.

\section{Concluding Remarks and Future Directions}

Based on the consideration of the jump risk of financial asset prices, the credit risk of the counterparty and the long-range dependence of financial asset prices in options trading, a more realistic pricing model for European vulnerable options is proposed in this paper. Assuming that the underlying asset price and the firm value of the counterparty are both governed by jump-diffusion processes under the bifractional Brownian motion environment, in which these jump-diffusion processes are correlated and have common factors in jump process components, the total jumps in the two processes are divided into idiosyncratic jumps for every asset price and common ones that affect the prices of all assets. In our framework, we apply the actuarial approach to derive the closed-form formulae for the European vulnerable options, which encompass many existing formulae as special cases.

This study also suggests several important directions for further research. First, it is rather interesting and meaningful to make empirical study for vulnerable options which could help to adjust the parameters in the pricing model to better capture the real data. Second is to take more practical assumptions into account based on the current model which would make the pricing results for vulnerable options accurate. For example, a pricing model for European vulnerable options under BFBM with stochastic interest rate, stochastic volatility, and jumps can be considered due to a number of empirical lines of evidence that the interest rate has the property of mean-reversion and constant volatility cannot capture the volatility smile or skew which can be captured by stochastic volatility models. Third, our model does not consider the constant periods of asset prices, which makes the application of the pricing model limited. Motivated by the aforementioned models in references [35-41] and the references therein, it is a good idea to construct a new pricing model for options under BFBM in subdiffusive regime.

\section{Data Availability}

The data used to support the findings of this research are available from the corresponding author upon request.

\section{Conflicts of Interest}

The authors declare no conflicts of interest.

\section{Acknowledgments}

This research was supported by the National Natural Science Foundation of China under Grant (72071130) and the Natural Science Foundation of Universities of Anhui Province (KJ2018A0429).

\section{References}

[1] F. Black and M. Scholes, "The pricing of options and corporate liabilities," Journal of Political Economy, vol. 81, no. 3, pp. 637-654, 1973.

[2] E. F. Fama, "Efficient capital markets: a review of the theory and empirical work," The Journal of Finance, vol. 25, no. 2, pp. 383-417, 1971.

[3] R. C. Merton, "Option pricing when underlying stock returns are discontinuous," Journal of Financial Economics, vol. 3, no. 1-2, pp. 125-144, 1976. 
[4] R. A. Jarrow and E. R. Rosenfeld, "Jump risks and the intertemporal capital asset pricing model," The Journal of Business, vol. 57, no. 3, pp. 337-351, 1984.

[5] C. A. Ball and W. N. Torous, "On jumps in common stock prices and their impact on call option pricing," The Journal of Finance, vol. 40, no. 1, pp. 155-173, 1985.

[6] S. G. Kou, "A jump-diffusion model for option pricing," Management Science, vol. 48, no. 8, pp. 1086-1101, 2002.

[7] M. R. Rodrigo, "Pricing of barrier options on underlying assets with jump-diffusion dynamics: a Mellin transform approach," Mathematics, vol. 8, pp. 1-20, 2020.

[8] A. Lipton, "Assets with jumps," Risk Magazine, vol. 15, no. 9, pp. 149-153, 2002.

[9] M. Jeannin and M. Pistorius, "A transform approach to compute prices and Greeks of barrier options driven by a class of Lévy processes," Quantitative Finance, vol. 10, no. 6, pp. 629-644, 2010.

[10] T. R. Li and M. R. Rodrigo, "Alternative results for option pricing and implied volatility in jump-diffusion models using Mellin transforms," European Journal of Applied Mathematics, vol. 28, pp. 1-38, 2016.

[11] M. R. Rodrigo and J. Goard, "Pricing of general European options on discrete dividend-paying assets with jump-diffusion dynamics," Applied Mathematical Modelling, vol. 64, pp. $47-54,2018$.

[12] R. C. Merton, "On the pricing of corporate debt: the risk structure of interest rates," The Journal of Finance, vol. 29, no. 2, pp. 449-470, 1974.

[13] R. A. Jarrow and S. M. Turnbull, "Pricing derivatives on financial securities subject to credit risk," The Journal of Finance, vol. 50, no. 1, pp. 53-85, 1995.

[14] H. Johnson and R. Stulz, "The pricing of options with default risk," The Journal of Finance, vol. 42, no. 2, pp. 267-280, 1987.

[15] P. Klein, "Pricing black-scholes options with correlated credit risk," Journal of Banking \& Finance, vol. 20, no. 7, pp. 1211-1229, 1996.

[16] P. Klein and M. Inglis, "Valuation of European options subject to financial distress and interest rate risk," The Journal of Derivatives, vol. 6, no. 3, pp. 44-56, 1999.

[17] M.-K. Lee, S.-J. Yang, and J.-H. Kim, "A closed form solution for vulnerable options with Heston's stochastic volatility," Chaos, Solitons \& Fractals, vol. 86, pp. 23-27, 2016.

[18] L. Tian, G. Wang, X. Wang, and Y. Wang, "Pricing vulnerable options with correlated credit risk under jump-diffusion processes," Journal of Futures Markets, vol. 34, no. 10, pp. 957-979, 2014.

[19] X. Wang, "Pricing vulnerable options with stochastic default barriers," Finance Research Letters, vol. 19, pp. 305-313, 2016.

[20] J. H. Yang, S. W. Zhou, H. T. Zhou, and K. Q. Guo, "Pricing vulnerable option under jump-diffusion model with incomplete information," Discrete Dynamics in Nature and Society, vol. 2019, Article ID 5848375, 8 pages, 2019.

[21] H. Niu, Y. Xing, and Y. Zhao, "Pricing vulnerable European options with dynamic correlation between market risk and credit risk," Journal of Management Science and Engineering, vol. 5, no. 2, pp. 125-145, 2020.

[22] S.-L. Liao and H.-H. Huang, "Pricing black-scholes options with correlated interest rate risk and credit risk: an extension," Quantitative Finance, vol. 5, no. 5, pp. 443-457, 2005.

[23] J.-H. Yoon and J.-H. Kim, "The pricing of vulnerable options with double Mellin transforms," Journal of Mathematical Analysis and Applications, vol. 422, no. 2, pp. 838-857, 2015.

[24] H. Niu and D. Wang, "Pricing vulnerable options with correlated jump-diffusion processes depending on various states of the economy," Quantitative Finance, vol. 16, no. 7, pp. 1129-1145, 2016.

[25] G. Wang, X. Wang, and K. Zhou, "Pricing vulnerable options with stochastic volatility," Physica A: Statistical Mechanics and Its Applications, vol. 485, pp. 91-103, 2017.

[26] M.-K. Lee and J.-H. Kim, "Pricing of defaultable options with multiscale generalized Heston's stochastic volatility," Mathematics and Computers in Simulation, vol. 144, pp. 235-246, 2018.

[27] C. Ma, Z. Ma, and S. Xiao, “A closed-form pricing formula for vulnerable European options under stochastic yield spreads and interest rates," Chaos, Solitons \& Fractals, vol. 123, pp. 59-68, 2019.

[28] X. Han, "Valuation of vulnerable options under the double exponential jump model with stochastic volatility," Probability in the Engineering and Informational Sciences, vol. 33, no. 1, pp. 81-104, 2019.

[29] P. Pasricha and A. Goel, "Pricing vulnerable power exchange options in an intensity based framework," Journal of Computational and Applied Mathematics, vol. 355, pp. 106-115, 2019.

[30] W. Xu, W. Xu, and W. Xiao, "Pricing black-scholes options with correlated credit risk and jump risk," Applied Economics Letters, vol. 22, no. 2, pp. 87-93, 2015.

[31] J. Janczura and A. Wyłomańska, "Subdynamics of financial data from fractional Fokker-Planck equation," Acta Physica Polonica B, vol. 40, no. 5, pp. 1341-1351, 2009.

[32] A. W. Lo, "Long-term memory in stock market prices," Econometrica, vol. 59, no. 5, pp. 1279-1313, 1991.

[33] D. O. Cajueiro and B. M. Tabak, "Long-range dependence and multifractality in the term structure of LIBOR interest rates," Physica A: Statistical Mechanics and Its Applications, vol. 373, pp. 603-614, 2007.

[34] S. H. Kang and S.-M. Yoon, "Long memory properties in return and volatility: evidence from the Korean stock market," Physica A: Statistical Mechanics and Its Applications, vol. 385, no. 2, pp. 591-600, 2007.

[35] M. Magdziarz, "Black-scholes formula in subdiffusive regime," Journal of Statistical Physics, vol. 136, no. 3, pp. 553-564, 2009.

[36] E. Barkai, R. Metzler, and J. Klafter, "From continuous time random walks to the fractional Fokker-Planck equation," Physical Review E, vol. 61, no. 1, pp. 132-138, 2000.

[37] R. Metzler and J. Klafter, "The random walk's guide to anomalous diffusion: a fractional dynamics approach," Physics Reports, vol. 339, no. 1, pp. 1-77, 2000.

[38] M. Magdziarz, A. Weron, and K. Weron, "Fractional FokkerPlanck dynamics: stochastic representation and computer simulation," Physical Review. E, Statistical, Nonlinear, and Soft Matter Physics, vol. 75, Article ID 016708, 2007.

[39] F. Shokrollahi, A. Kılıçman, and M. Magdziarz, "Pricing European options and currency options by time changed mixed fractional Brownian motion with transaction costs," International Journal of Financial Engineering, vol. 3, no. 1, Article ID 1650003, 2016.

[40] C. Li, Option Pricing with Generalized Continuous Time Random Walk Models, Queen Mary University of London, London, UK, 2016.

[41] M. Magdziarz and J. Gajda, "Anomalous dynamics of BlackCScholes model time-changed by inverse subordinators," Acta Physica Polonica B, vol. 43, no. 5, pp. 1093-1110, 2012.

[42] J. H. Jeon and R. Metzler, "Fractional Brownian motion and motion governed by the fractional Langevin equation in 
confined geometries," Physical Review. E, Statistical, Nonlinear, and Soft Matter Physics, vol. 81, Article ID 021103, 2010.

[43] E. E. Peters, "Fractal structure in the capital markets," Financial Analysts Journal, vol. 7, no. 32, pp. 434-453, 1989.

[44] L. C. G. Rogers, "Arbitrage with fractional brownian motion," Mathematical Finance, vol. 7, no. 1, pp. 95-105, 1997.

[45] P. Cheridito, "Arbitrage in fractional brownian motion models," Finance and Stochastics, vol. 7, no. 4, pp. 533-553, 2003.

[46] T. Björk and H. Hult, "A note on wick products and the fractional Black-Scholes model," Finance and Stochastics, vol. 9, no. 2, pp. 197-209, 2005.

[47] C. Houdré and J. Villa, "An example of infinite dimensional quasi-helix," Contemporary Mathematics, vol. 366, pp. 195201, 2003.

[48] F. Russo and C. A. Tudor, "On bifractional Brownian motion," Stochastic Processes and Their Applications, vol. 116, no. 5, pp. 830-856, 2006.

[49] K. Es-Sebaiy and C. Tudor, "Multidimentsional bifractional Brownian motion: itô and Tanakás formulas," Stochastics and Dynamics, vol. 7, no. 3, pp. 365-388, 2007.

[50] M. Lifshits and K. Volkova, "Bifractional Brownian motion: existence and border cases," ESAIM: Probability and Statistics, vol. 19, pp. 766-781, 2015.

[51] F. Xu, "Bifractional black-scholes model for pricing European options and compound options," Journal of Systems Science and Information, vol. 8, no. 4, pp. 346-355, 2020.

[52] C. A. Tudor and Y. M. Xiao, "Sample path properties of bifractional Brownian motion,” Bernoulli, vol. 13, pp. 10231052, 2007.

[53] P. Lei and D. Nualart, "A decomposition of the bifractional Brownian motion and some applications," Statistics and Probability Letters, vol. 79, no. 5, pp. 619-624, 2008.

[54] X. Bardina and K. Es-Sebaiy, "An extension of bifractional Brownian motion," Communications on Stochastic Analysis, vol. 5, no. 2, pp. 333-340, 2011.

[55] M. El Machkouri, K. Es-Sebaiy, and Y. Ouknine, "Least squares estimator for non-ergodic Ornstein-Uhlenbeck processes driven by Gaussian processes," Journal of the Korean Surgical Society, vol. 45, no. 3, pp. 329-341, 2016.

[56] M. Ait Ouahra, H. Ouahhabi, and A. Sghir, "Continuity in law of some additive functionals of bifractional Brownian motion," Stochastics, vol. 91, no. 4, pp. 613-628, 2019.

[57] M. Bladt and T. H. Rydberg, "An actuarial approach to option pricing under the physical measure and without market assumptions," Insurance: Mathematics and Economics, vol. 22, no. 1, pp. 65-73, 1998.

[58] H. F. Yan and S. Y. Liu, "New method to option pricing for the general Black-Scholes model: an acturarial approach," Applied Mathematics and Mechanics, vol. 24, pp. 730-738, 2003.

[59] A. Falkowski, "Actuarial approach to option pricing in a fractional black-scholes model with time-dependent volatility," Bulletin of the Polish Academy of Sciences Mathematics, vol. 61 , no. 2, pp. 181-193, 2013.

[60] F. Shokrollahi and A. Klllçman, "Actuarial approach in a mixed fractional Brownian motion with jumps environment for pricing currency option," Advances in Difference Equations, vol. 2015, no. 1, pp. 1-8, 2015.

[61] H. Xue and Q. Li, "An actuarial approach to the minimum or maximum option pricing in fractional brownian motion environment," in Proceedings of the 2010 2nd IEEE International Conference on Information and Financial Engineering (ICIFE), Chongqing, China, September 2010.
[62] J.-R. Liang, J. Wang, L.-J. Lǔ, H. Gu, W.-Y. Qiu, and F.-Y. Ren, "Fractional fokker-planck equation and black-scholes formula in composite-diffusive regime," Journal of Statistical Physics, vol. 146, no. 1, pp. 205-216, 2012.

[63] J. Liu, L. Yan, and C. Ma, "Pricing options and convertible bonds based on an actuarial approach," Mathematical Problems in Engineering, vol. 2013, Article ID 676148, 9 pages, 2013.

[64] A. Falkowski, L. Słomiński, and B. Ziemkiewicz, "Weak and strong discrete-time approximation of fractional SDEs," Lithuanian Mathematical Journal, vol. 54, no. 4, pp. 409-428, 2014. 